Geometry $\&$ Topology

Volume 1 (1997) 91-109

Published: 21 December 1997

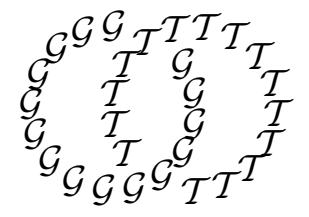

\title{
Finiteness of Classifying Spaces of Relative Diffeomorphism Groups of 3-Manifolds
}

\author{
Allen Hatcher \\ DARryl McCullough \\ Department of Mathematics, Cornell University \\ Ithaca, NY 14853, USA \\ and \\ Department of Mathematics, University of Oklahoma \\ Norman, OK 73019, USA \\ Email: hatcher@math.cornell.edu and dmccullough@math.ou.edu
}

\begin{abstract}
The main theorem shows that if $M$ is an irreducible compact connected orientable 3-manifold with non-empty boundary, then the classifying space $B D i f f(M$ rel $\partial M)$ of the space of diffeomorphisms of $M$ which restrict to the identity map on $\partial M$ has the homotopy type of a finite aspherical $\mathrm{CW}$-complex. This answers, for this class of manifolds, a question posed by M Kontsevich. The main theorem follows from a more precise result, which asserts that for these manifolds the mapping class group $\mathcal{H}(M$ rel $\partial M)$ is built up as a sequence of extensions of free abelian groups and subgroups of finite index in relative mapping class groups of compact connected surfaces.
\end{abstract}

AMS Classification numbers Primary: 57M99

Secondary: 55R35, 58D99

Keywords: 3-manifold, diffeomorphism, classifying space, mapping class group, homeotopy group, geometrically finite, torsion

Proposed: Robion Kirby

Seconded: Joan Birman, David Gabai
Received: 12 June 1997

Revised: 19 December 1997

Copyright Geometry and Topology 
For a compact connected 3-manifold $M$, let Diff $(M$ rel $R$ ) denote the group of diffeomorphisms $M \rightarrow M$ restricting to the identity on the subset $R$. We give $\operatorname{Diff}\left(M\right.$ rel $R$ ) the $C^{\infty}$-topology, as usual. M. Kontsevich has conjectured (problem 3.48 in [12]) that the classifying space BDiff ( $M$ rel $\partial M$ ) has the homotopy type of a finite complex when $\partial M$ is non-empty. In this paper we prove the conjecture for irreducible orientable $3-$ manifolds. In fact, more is true in this case.

Main Theorem Let $M$ be an irreducible compact connected orientable 3manifold and let $R$ be a non-empty union of components of $\partial M$, including all the compressible ones. Then BDiff $(M$ rel $R$ ) has the homotopy type of an aspherical finite $C W$-complex.

Actually, the assertion that $B \operatorname{Diff}(M$ rel $R$ ) is aspherical has been known for some time (see $[2,3,6]$ ), as a special case of more general results about Haken manifolds. Thus the finiteness question for $\operatorname{BDiff}(M$ rel $R$ ) is equivalent to whether the mapping class group $\pi_{0}(\operatorname{Diff}(M$ rel $R))$, which we denote by $\mathcal{H}(M$ rel $R)$, is a group whose classifying space is homotopy equivalent to a finite complex. Such groups are called geometrically finite.

It is a standard elementary fact that a geometrically finite group must be torsion-free. Thus the Main Theorem implies that $\mathcal{H}(M$ rel $R)$ is torsion-free, a fact which can be deduced from [5]. If we drop the condition that the diffeomorphisms restrict to the identity on $R$, or allow $M$ to be closed, then $\mathcal{H}(M)$ can have torsion (for example if $M$ is a hyperbolic $3-$ manifold with non-trivial isometries), and thus $\operatorname{BDiff}(M)$ can be aspherical but not of the homotopy type of a finite complex. For all Haken 3-manifolds, however, there exist finite-sheeted covering spaces of $B D i f f(M)$ and BDiff $(M$ rel $R$ ) which have the homotopy type of a finite complex [13]. The Main Theorem can be viewed as a refinement of this result for the case of $B D i f f(M$ rel $R$ ).

If the irreducibility condition on $M$ is dropped, $B D i f f(M$ rel $R$ ) need no longer be aspherical. Indeed, its higher homotopy groups can be rather complicated, and in particular $\pi_{2}(B D i f f(M$ rel $R))$ is generally not finitely generated [10] This does not exclude the possibility that $B D i f f(M$ rel $R$ ) has the homotopy type of a finite complex (for example, $S^{1} \vee S^{2}$ is a finite complex having non-finitely generated $\pi_{2}$ ), and it would be very interesting to know whether Kontsevich's conjecture holds in these cases. If so, it would indicate that $\operatorname{BDiff}(M$ rel $R$ ) is more tractable than has generally been supposed.

The Main Theorem follows directly from a structural result about $\mathcal{H}(M$ rel $R)$ which we will state below. To place our result in historical context, and to 
review some of the ingredients that go into its proof, we will first survey some previous work on mapping class groups of Haken 3-manifolds. Recall that according to the basic structure theorem of Jaco-Shalen [7] and Johannson [8], a Haken manifold with incompressible boundary admits a characteristic decomposition into pieces which are either fibered (admitting an $I$-fibering or a Seifert fibering) or simple (admitting no incompressible torus or proper annulus which is not properly homotopic into the boundary). By the late 1970's, substantial information had been obtained about the mapping class groups of these pieces. For the pieces that are $I$-bundles, the mapping class group is essentially the same as the mapping class group of the quotient surface. The necessary technical results to analyze this case are contained in Waldhausen's seminal paper [16]. For a Seifert-fibered piece $W$, the analysis was due to Waldhausen (pages 85-86 of [16], page 36 of [17]) and Johannson (proposition 25.3 of [8]). After showing that one can restrict attention to diffeomorphisms preserving the fiber structure, they deduced that $\mathcal{H}(W)$ fits into a short exact sequence where the kernel group is finitely-generated abelian and the quotient group is a surface mapping class group. For the simple pieces, Johannson proved that the group of mapping classes preserving the frontier is finite (proposition 27.1 of [8]). Of course, this was carried out without reference to the hyperbolic structure later discovered to exist on these pieces. Today, this finiteness is often viewed as a consequence of Mostow rigidity, which implies that if $W$ is a 3-manifold with a complete hyperbolic structure with finite volume, then $\operatorname{Out}\left(\pi_{1}(W)\right)$ is finite, and from Waldhausen's fundamental work, this group is isomorphic to the mapping class group. However, when $\partial M$ has components other than tori, the simple pieces of $M$ might not admit hyperbolic structures of finite volume, and indeed their mapping class groups may not be finite, but their groups of mapping classes preserving the frontier will be finite.

By combining the information on these two types of pieces, Johannson proved the first general result on mapping class groups of Haken 3-manifolds. This result, corollary 27.6 in [8], says that in the case when $M$ has incompressible boundary, the subgroup of mapping classes generated by Dehn twists about tori and properly imbedded annuli in $M$ has finite index in $\mathcal{H}(M)$. (For a definition of Dehn twist, see section 3 below.)

At about the same time, techniques for controlling isotopies between diffeomorphisms of 3-manifolds were being developed, leading to more refined structural details about mapping class groups. Laudenbach [9] proved that (apart from a few easily-understood exceptions) an isotopy between two diffeomorphisms of a Haken 3-manifold that preserve an incompressible surface can be deformed to an isotopy that preserves the surface at each level of the isotopy. This

\section{Geometry and Topology, Volume 1 (1997)}


was extended to parameterized families by Hatcher [3] and Ivanov [6], giving the key ingredient in the proof that when $\partial M$ is non-empty, the components of $\operatorname{Diff}(M$ rel $\partial M)$ are contractible, and consequently BDiff $(M$ rel $\partial M)$ is an aspherical complex. Laudenbach's result also led to the extension of Harer's homological finiteness results on 2-manifold mapping class groups to dimension 3 by McCullough [13].

Among Harer's results was the fact that 2-manifold mapping class groups contain geometrically finite subgroups of finite index. Using Harer's constructions, in the simplified exposition of [4], we strengthen this as follows.

Lemma 1.2 Let $S$ be a compact connected surface, and let $J$ and $K$ be 1-dimensional submanifolds of $\partial S$ with $J \cap K=\partial J \cap \partial K$. If $K$ is non-empty, then $\mathcal{H}(S, J$ rel $K)$ is geometrically finite.

Here, the notation $\mathcal{H}(S, J$ rel $K)$ indicates the mapping classes that carry $J$ diffeomorphically to $J$. The geometric finiteness of $\mathcal{H}(M$ rel $R)$ is then an immediate consequence of the following structure theorem, whose proof occupies most of this paper.

Filtration Theorem Let $M$ be an irreducible compact connected orientable 3-manifold and let $R$ be a non-empty union of components of $\partial M$, including all the compressible ones. Then there is a filtration

$$
0=G_{0} \subset G_{1} \subset \cdots \subset G_{n}=\mathcal{H}(M \text { rel } R)
$$

with each $G_{i}$ a normal subgroup of $G_{i+1}$ such that $G_{i+1} / G_{i}$ is either a finitely generated free abelian group or a subgroup of finite index in a mapping class group $\mathcal{H}(S, J$ rel $K)$ of a compact connected surface $S$.

Let us describe how the filtration arises. An argument using a family of compressing discs for the compressible components of $\partial M$ reduces the proof to the case that $\partial M$ is incompressible. Then, each mapping class contains representatives which preserve the characteristic decomposition of Jaco-Shalen and Johannson, and Laudenbach's result implies that isotopies between such diffeomorphisms can also be assumed to preserve the decomposition. We can filter $M$ by an increasing sequence of submanifolds $V_{i}$, each obtained from the preceding one by attaching one of the characteristic pieces. This leads to a decreasing filtration of $\mathcal{H}(M$ rel $R)$ by the subgroups represented by diffeomorphisms which restrict to the identity on succesively larger $V_{i}$ 's. The preceding remarks imply that the successive quotients for this filtration are subgroups

Geometry and Topology, Volume 1 (1997) 
of relative mapping class groups of the corresponding attached characteristic pieces. This reduces us to understanding the relative mapping class groups of the characteristic pieces.

In the case of fibered pieces, the analysis is that of Waldhausen and Johannson. We present it in the relative cases that we will need as lemmas 2.1 and 2.2. The upshot is that after interpolating one more stage in the filtration, the two resulting quotient groups are of the desired types. For the simple pieces, the finiteness result of Johannson implies that the relative mapping class group has a finitely-generated abelian subgroup of finite index, generated by Dehn twists along peripheral tori and annuli. But to rule out the existence of torsion, we show that the relative mapping class group itself is finitely-generated free abelian. This is done using an appropriate form of Mostow rigidity. In the case that $\partial M$ consists entirely of tori, the usual form of Mostow rigidity suffices, but in the general case an extended version is needed. To state this, let $W$ be a compact connected orientable irreducible 3-manifold with non-empty boundary, let $T$ be the union of its torus boundary components, and let $A$ be a union of disjoint incompressible annuli in $\partial W-T$. Certain assumptions, listed at the beginning of section 3, are made which are satisfied when $W$ is a simple piece of the characteristic decomposition of a Haken 3 -manifold and $A$ is the union of the components of the frontier of $W$ that are annuli. Let $\operatorname{Diff}(W, A)$ denote the diffeomorphisms of $W$ that take $A$ diffeomorphically to $A$, and let $\mathcal{H}(W, A)=\pi_{0}(\operatorname{Diff}(W, A))$.

Lemma 3.1 $W-(A \cup T)$ has a hyperbolic structure with totally geodesic boundary. Its group of isometries $\operatorname{Isom}(W-(A \cup T))$ is finite, and $\operatorname{Isom}(W-$ $(A \cup T)) \rightarrow \mathcal{H}(W, A)$ is an isomorphism.

This version of Mostow Rigidity is folklore, but as will be seen, it is not such a simple matter to give a real proof. A key ingredient is a theorem of Tollefson [15], which provides a very strong uniqueness statement for certain involutions of Haken 3-manifolds.

\section{Preliminaries}

In this section we collect some auxiliary results that will be used in the proof of the Main Theorem.

As explained in the introductory section, the Filtration Theorem will show that $\mathcal{H}(M$ rel $R)$ is built up by a sequence of extensions of geometrically finite groups. Then, the following lemma will imply the Main Theorem.

Geometry and Topology, Volume 1 (1997) 
Lemma 1.1 Let $1 \rightarrow H \rightarrow G \rightarrow K \rightarrow 1$ be a short exact sequence of groups. If $H$ and $K$ are geometrically finite, then $G$ is geometrically finite.

Proof By proposition 5(c) of [14], a group $\Gamma$ is geometrically finite if and only if it is finitely presented and FL. The latter means that there is a finite length resolution of the trivial $\mathbb{Z} \Gamma$-module $\mathbb{Z}$ by finitely generated free $\mathbb{Z} \Gamma$-modules. By proposition 6(b) of [14], extensions of FL groups are FL, and the lemma follows.

The next lemma provides geometrically finite groups that will form some of the building blocks for BDiff ( $M$ rel $R$ ).

Lemma 1.2 Let $S$ be a compact connected surface, and let $J$ and $K$ be $1-$ dimensional submanifolds of $\partial S$ with $J \cap K=\partial J \cap \partial K$. If $K$ is non-empty, then $\mathcal{H}(S, J$ rel $K)$ is geometrically finite.

Proof We use constructions due to Harer, in the simplified exposition of [4]. We may assume that $J$ consists of arcs, since if $J^{\prime}$ consists of the arc components of $J$, then $\mathcal{H}(S, J$ rel $K)$ has finite index in $\mathcal{H}\left(S, J^{\prime}\right.$ rel $\left.K\right)$. As a further simplification, we may assume all the components of $K$ are circles, since replacing each arc of $K$ by the component of $\partial S$ containing it, and deleting from $J$ any components engulfed by $K$ in this process, has no effect on $\mathcal{H}(S, J$ rel $K)$.

Let $P$ be the finite set obtained by choosing one point from each circle of $K$ and one point from the interior of each arc of $J$. Consider finite systems of arcs in $S$ with endpoints in $P$ and with interiors disjointly embedded in the interior of $S$, such that:

(a) Each arc is essential: cutting $S$ along the arc does not produce two components, one of which is a disc. This is equivalent to saying that the arc represents a non-trivial element of $\pi_{1}(S, \partial S)$.

(b) No two arcs in a system are isotopic in $S$ rel endpoints.

If $S$ is a disc, or annulus for which $J \cup K$ meets only one component of $\partial S$, then $\mathcal{H}(S, J$ rel $K)$ is trivial and the lemma holds. Otherwise, form a simplicial complex $\mathcal{A}$ whose $k$-simplices are the isotopy classes of systems of $k+1$ arcs satisfying (a) and (b). The barycentric subdivision $\mathcal{A}^{\prime}$ of $\mathcal{A}$ is the simplicial complex associated to the partially ordered set of isotopy classes of arc systems, with the partial ordering given by inclusion of systems. We are interested in the subcomplex $\mathcal{B} \subset \mathcal{A}^{\prime}$ associated to the partially ordered set of systems 
whose complementary components are either discs or once-punctured discs, the puncture being a component of $\partial S$ that does not meet $J \cup K$. The proof in [4] describes a surgery process that determines a flow on $\mathcal{A}$ that moves $\mathcal{A}$ into the star of a vertex corresponding to a single arc. This flow preserves $\mathcal{B}$. If the process is successively repeated for the arcs that make up a vertex of $\mathcal{B}$, then $\mathcal{B}$ will be moved into the star of that vertex, hence can be further contracted to the vertex moving only through $\mathcal{B}$. Thus $\mathcal{B}$ is also contractible.

The group $\mathcal{H}(S, J$ rel $K)$ acts simplicially on $\mathcal{A}$ and $\mathcal{B}$. The action on $\mathcal{B}$ is without fixed points. For if a point in a simplex of $\mathcal{B}$ were fixed by an element of $\mathcal{H}(S, J$ rel $K)$, the simplex would be invariant, hence fixed since its vertex arc systems are distinguished from each other by the number of arcs they contain. Thus one would have an arc system $\alpha$, representing a vertex of $\mathcal{B}$, which is taken by an element $h \in \operatorname{Diff}(S, J$ rel $K)$ to an isotopic arc system $h(\alpha)$. By isotopy extension, we may assume $h(\alpha)=\alpha$. The defining property of $\mathcal{B}$ implies that each component of $S-\alpha$ is either a disc or a disc with a puncture corresponding to a component of $\partial S$ that does not meet $J \cup K$. Since $K$ is non-empty, $h$ must preserve the closure of at least one of the components of $S-\alpha$ and fix all elements of $P$ that it contains. By induction on the number of components of $S-\alpha$, we deduce that $h$ must fix each point of $P$ and preserve each component of $S-\alpha$. It follows that $h$ is isotopic, relative to $K$ and preserving $J$, to the identity of $S$.

Thus the quotient $\mathcal{B} / \mathcal{H}(S, J$ rel $K)$ is a $K(\mathcal{H}(S, J$ rel $K), 1)$. This quotient is a finite complex since arc systems fall into finitely many orbits under the action of $\operatorname{Diff}(S, J$ rel $K)$.

We will use the following consequence of a theorem of Laudenbach.

Lemma 1.3 Let $M$ be a compact connected irreducible 3-manifold which does not contain two-sided projective planes. In $M$ let $F$ be a properly imbedded 2-sided incompressible 2-manifold, no component of which is a 2-sphere. Let $J_{t}: M \rightarrow M$ be an isotopy such that $J_{0}$ is the identity and $J_{1}(F)=F$. If either

(i) $\partial F$ is non-empty and $J_{t}(\partial F)=\partial F$ for all $t$, or

(ii) $M$ does not fiber over $S^{1}$ with $F$ as fiber,

then $J_{t}$ is deformable (through isotopies and relative to $M \times \partial I$ ) to an isotopy which preserves $F$ at each level. In case (i), or in case (ii) when $F$ is closed, the deformation can also be taken relative to $\partial M \times I$.

Geometry and Topology, Volume 1 (1997) 
Proof By induction we may assume that $F$ is connected. Fix a basepoint $x_{0}$ in the interior of $F$. The proof of theorem 7.1 of [16] shows that under hypothesis (i) or (ii), $J_{t}$ is deformable relative to $M \times \partial I$ to a homotopy $H_{t}$ which preserves $F$ at each level, and also shows that $\left.J_{1}\right|_{F}$ must be an orientationpreserving diffeomorphism of $F$. Let $h_{t}$ be the restriction of $H_{t}$ to $F$. Any homotopy from the identity map to an orientation-preserving diffeomorphism of $F$ can be deformed relative to $F \times I$ to an isotopy [1], that is, there exists an isotopy $h_{t}^{\prime}$ from the identity of $F$ to $h_{1}$, such that the path $h_{t}$ followed by the reverse of $h_{t}^{\prime}$ is a contractible loop in the space of homotopy equivalences of $F$. Let $K_{t}$ be the isotopy of $M$ obtained by extension of the reverse of $h_{t}^{\prime}$, starting from $J_{1}$, and let $L$ be the product isotopy $J K$. Then $L$ has trivial trace at $x_{0}$, and $L_{1}$ is the identity on $F$. Since $\operatorname{Diff}(M \operatorname{rel} \partial M) \rightarrow \operatorname{Imb}\left(x_{0}, M-\partial M\right)$ is a fibration, $L$ is deformable relative to $\partial M \times I$ to an isotopy that fixes $x_{0}$.

Let $\ell_{t}$ be the restriction of $L_{t}$ to $F$. What is proven on pages 49-62 of [9] (see the comments at the end of page 48) is that $\pi_{1}\left(\operatorname{Imb}\left(F, M\right.\right.$ rel $\left.\left.x_{0}\right)\right)=0$. So $\ell_{t}$ is deformable to the constant loop at the inclusion. Since $\operatorname{Diff}(M) \rightarrow \operatorname{Imb}(F, M)$ is a fibration, this deformation of $\ell_{t}$ extends to a deformation of $L_{t}$ to an isotopy which is the identity on $F$ for every $t$. Since $K$ preserves $F$ at every level, it follows that $J$ is deformable to an isotopy $J^{\prime}$ which preserves $F$ at every level. When $F$ is closed, all deformations can be taken relative to $\partial M \times I$. In case (i), the trace of $J$ at a point in $\partial F$ is a path in $\partial F$. Let $G$ be a component of $\partial M$. Using [1], and the fact that $G$ is not the $2-$ sphere, any two paths in $\operatorname{Diff}(G)$ with the same trace and the same endpoints are deformable to each other. Therefore $J^{\prime}$ can be deformed to agree with $J$ on $\partial M \times I$. Since $\pi_{2} \operatorname{Diff}(G)=0$, the deformation from $J$ to $J^{\prime}$ can then be taken relative to $\partial M \times I$.

\section{$2 \quad$ Fibered Manifolds}

In this section $M$ will be a compact connected orientable 3 -manifold whose boundary is decomposed as the union of two compact subsurfaces $A$ and $B$ which intersect only in the circles of $\partial A=\partial B$. We assume that the components of $A$ are annuli. We shall be considering $\operatorname{Diff}(M, A$ rel $R)$, the group of diffeomorphisms of $M$ taking $A$ to itself and restricting to the identity on $R$, a non-empty union of components of $A$ and $B$.

Suppose first that $M$ is an $I$-bundle over a compact connected surface $S$, with projection map $p: M \rightarrow S$. We let $A$ be the union of the fibers over $\partial S$, so $B$ is the associated $\partial I$-bundle.

Geometry and Topology, Volume 1 (1997) 
Lemma 2.1 If $S$ has negative Euler characteristic, then $\mathcal{H}(M, A$ rel $R)$ is isomorphic to $\mathcal{H}(S$ rel $p(R))$. In particular, $\mathcal{H}(M, A$ rel $R)=0$ if $R$ is not contained in $A$.

Proof We assert that the inclusion $\operatorname{Diff}_{f}(M, A$ rel $R) \hookrightarrow \operatorname{Diff}(M, A$ rel $R)$ of the subgroup consisting of diffeomorphisms taking fibers to fibers induces an isomorphism on $\pi_{0}$. In the case when $R$ contains at most one component of $B$, this follows from corollary 5.9 of [8]. Otherwise, $M$ is a product $I$-bundle and $B$ has two components. Let $R^{\prime}=\overline{R-L}$ where $L$ is one of the components of $B$. Then the assertion holds for $\operatorname{Diff}\left(M, A\right.$ rel $\left.R^{\prime}\right)$ and shows that it is contractible. Since $L$ has negative Euler characteristic, the identity component $\operatorname{diff}\left(L\right.$ rel $\left.L \cap R^{\prime}\right)$ is contractible. Therefore the fibration $\operatorname{Diff}\left(M, A\right.$ rel $\left.R^{\prime}\right) \rightarrow$ $\operatorname{diff}\left(L\right.$ rel $\left.L \cap R^{\prime}\right)$ is a homotopy equivalence, so its fiber $\operatorname{Diff}(M, A$ rel $R)$ is contractible and the assertion holds in this case as well.

Now, viewing $M$ as the mapping cylinder of the projection $B \rightarrow S$, there is a subgroup of $\operatorname{Diff}_{f}(M$ rel $R$ ) consisting of diffeomorphisms taking each level $B \times\{t\}$ of the mapping cylinder to itself, and the inclusion of this subgroup also induces an isomorphism on $\pi_{0}$ since $\operatorname{Diff}(I$ rel $\partial I$ ) is contractible and $R$ is non-empty. Diffeomorphisms in this subgroup are determined by the quotient diffeomorphism they induce on $S$, and the result follows.

Suppose now that $M$ is an orientable compact connected irreducible 3-manifold Seifert fibered over the surface $S$, with projection $p: M \rightarrow S$. We assume the annuli of $A$ in the decomposition $\partial M=A \cup B$ are unions of fibers.

The images of the exceptional fibers form a finite set of exceptional points $E \subset$ $S-\partial S$. Each exceptional point can be labelled by a rational number normalized to lie in the interval $(0,1)$, describing the local structure of the Seifert fibering near the corresponding exceptional fiber of $M$. Let $\operatorname{Diff}^{*}(S, E \cup p(A)$ rel $p(R))$ be the subgroup of $\operatorname{Diff}(S, p(A)$ rel $p(R))$ consisting of diffeomorphisms permuting the points of $E$ in such a way as to preserve the labelling, and let $\mathcal{H}^{*}(S, E \cup p(A)$ rel $p(R))$ denote the corresponding mapping class group.

Lemma 2.2 There is a split short exact sequence

$$
0 \rightarrow H_{1}(S, \partial S-p(R) ; \mathbb{Z}) \rightarrow \mathcal{H}(M, A \text { rel } R) \rightarrow \mathcal{H}^{*}(S, E \cup p(A) \operatorname{rel} p(R)) \rightarrow 0 .
$$

Proof This is similar to section 25 of [8]. Denote by $\operatorname{Diff}_{f}(M, A$ rel $R)$ the subgroup of Diff $(M, A$ rel $R$ ) consisting of diffeomorphisms taking fibers to fibers. The first assertion is that the inclusion of $\operatorname{Diff}_{f}(M, A$ rel $R$ ) into 
$\operatorname{Diff}(M, A$ rel $R)$ induces an isomorphism on $\pi_{0}$. A proof of this without the "rel $R$ " is indicated on pages $85-86$ of [16], and the same proof works rel $R$.

Since elements of $\operatorname{Diff}_{f}(M, A$ rel $R)$ take exceptional fibers to exceptional fibers with the same labeling data, a natural homomorphism $\Phi: \operatorname{Diff}_{f}(M, A$ rel $R) \rightarrow$ $\operatorname{Diff}^{*}(S, E \cup p(A)$ rel $p(R))$ is induced by projection. By theorem 8.3 of [11], $\Phi$ is locally trivial, so is a Serre fibration. (One can also check directly that $\Phi$ is a Serre fibration. Since we are dealing with groups, it suffices to construct a $k$-parameter isotopy of the identity of $M$ which lifts a given $k$-parameter isotopy of the identity of $S$, and this is not difficult.) A section of $\Phi$ can be constructed as follows. Let $M_{0}$ be $M$ with an open fibered tubular neighborhood of the exceptional fibers deleted, and let $S_{0}$ be the image of $M_{0}$ in $S$. If $S$ is orientable, then $M_{0}$ is a product $S_{0} \times S^{1}$, from which $M$ can be obtained by filling in solid torus neighborhoods of the exceptional fibers in a standard way depending only on the labeling data of the exceptional fibers. Diffeomorphisms of $S_{0}$ give rise to diffeomorphisms of $M_{0}$ by taking the product with the identity on $S^{1}$, and then these diffeomorphisms extend over $M$ in the obvious way, assuming the labeling data is preserved. In case $S$ is non-orientable, $M_{0}$ can be obtained by doubling the mapping cylinder of the orientable double cover $\widetilde{S}_{0} \rightarrow S_{0}$. Diffeomorphisms of $S_{0}$ lift canonically to diffeomorphisms of $\widetilde{S}_{0}$, hence by taking the induced diffeomorphisms of mapping cylinders we get a section of $\Phi$ in this case too.

Thus from the exact sequence of homotopy groups of the fibration $\Phi$ we obtain the split short exact sequence of the Proposition but with $H_{1}(S, \partial S-p(R))$ replaced by $\pi_{0}(X)$ where $X$ is the fiber of $\Phi$. It remains then to produce an isomorphism $\pi_{0}(X) \cong H_{1}(S, \partial S-p(R))$ (cf lemma 25.2 of [8]).

The fiber $X$ of $\Phi$ consists of the diffeomorphisms taking each circle fiber of $M$ to itself. Note that orientations of fibers are preserved since we are considering only diffeomorphisms which are the identity on $R$. We may assume elements of $X$ restrict to rotations of each circle fiber, in view of the fact that the groups of orientation-preserving diffeomorphisms of $S^{1}$ has the homotopy type of the rotation subgroup. There is no harm in pretending the exceptional fibers are not exceptional since the rotation of a exceptional fiber is determined by the rotations of nearby fibers. If $S$ is orientable, then $M=S \times S^{1}$ and diffeomorphisms which rotate fibers are the same as maps $(S, p(R)) \rightarrow\left(S^{1}, 1\right)$, measuring the angle of rotation in each fiber. Thus $\pi_{0}(X)$ is the group of homotopy classes of maps $(S, p(R)) \rightarrow\left(S^{1}, 1\right)$, ie $H^{1}(S, p(R))$, which is isomorphic to $H_{1}(S, \partial S-p(R))$ by duality. When $S$ is non-orientable one could presumably make the same sort of argument using cohomology with local coefficients, but 
instead we give a direct geometric argument, which applies when $S$ is orientable as well.

We can construct $S$ from a collar $p(R) \times I$ by attaching 1 -handles, plus a single 2 -handle if $p(R)=\partial S$. The core arcs of the 1-handles, extended through the collar to $p(R)$, lift to annuli $A_{i}$ in $M$ with $\partial A_{i} \subset \partial M$. Each diffeomorphism in $X$ restricts to a loop of diffeomorphisms of $S^{1}$ on each $A_{i}$. Since $\pi_{1}\left(\operatorname{Diff}\left(S^{1}\right)\right) \cong$ $\mathbb{Z}$, we thus have a homomorphism $\phi: \pi_{0}(X) \rightarrow \mathbb{Z}^{n}$ if there are $n A_{i}$ 's. Clearly $\phi$ is an injection, so $\pi_{0} X$ is finitely generated free abelian. If $p(R) \neq \partial S$, so there is no $2-$ handle, then $\phi$ is obviously surjective as well. This is also true if $p(R)=\partial S$, since it is not hard to see that Dehn twist diffeomorphisms of the $A_{i}$ 's extend to diffeomorphisms in $X$. A homology calculation shows that $H_{1}(S, \partial S-p(R))$ is a direct sum of $n$ copies of $\mathbb{Z}$ since $p(R)$ is non-empty.

Remark The group $\mathcal{H}^{*}(S, E \cup p(A)$ rel $p(R))$ is isomorphic to a subgroup of finite index in $\mathcal{H}\left(S_{0}, p(A)\right.$ rel $\left.p(R)\right)$, where $S_{0}$ is obtained from $S$ by deleting open discs about the points of $E$. According to lemma 1.2, the latter is geometrically finite. Since $H_{1}(S, \partial S-p(R) ; \mathbb{Z})$ is free abelian when $R$ is non-empty, lemma 2.2, together with lemma 2.3 below, implies the Main Theorem in the case when $M$ is a Seifert manifold.

The proof of our next lemma will use Dehn twists of $M$, which are diffeomorphisms defined as follows. Let $F$ be a torus or annulus, either properly imbedded in $M$ or contained in $\partial M$, and let $F \times I$ be a submanifold of $M$ with $F=F \times\{0\}$. For a loop $\gamma: I \rightarrow \operatorname{Diff}(F)$ representing an element of $\pi_{1}\left(\operatorname{Diff}(F), i d_{F}\right)$, a Dehn twist is defined by putting $h(y, t)=\left(\gamma_{t}(y), t\right)$ for $(y, t) \in F \times I$, and $h(x)=x$ for $x \notin F \times I$.

Lemma 2.3 Let $M, A$, and $R$ be as in lemma 2.1 or 2.2. Then for all $i>0$, $\pi_{i}(\operatorname{Diff}(M, A$ rel $R))=0$.

Proof Consider the restriction fibration

$$
\operatorname{Diff}(M \operatorname{rel} \partial M) \longrightarrow \operatorname{Diff}(M, A \text { rel } R) \stackrel{\rho}{\longrightarrow} \operatorname{Diff}(\partial M, A \text { rel } R) .
$$

For $i \geq 1, \pi_{i}(\operatorname{Diff}(M \operatorname{rel} \partial M))=0$ by [3]. For $i>1, \pi_{i}(\operatorname{Diff}(\partial M, A$ rel $R))=$ 0 by surface theory, so we need only check injectivity of the boundary homomorphism $\partial: \pi_{1}(\operatorname{Diff}(\partial M, A$ rel $R)) \rightarrow \pi_{0}(\operatorname{Diff}(M$ rel $\partial M))$. The only components of $\partial M$ which can contribute to $\pi_{1}(\operatorname{Diff}(\partial M, A$ rel $R))$ are torus components disjoint from $R$. Such a torus disjoint from $A$ contributes a $\mathbb{Z} \times \mathbb{Z}$ factor, while a torus which contains components of $A$ contributes a $\mathbb{Z}$ factor. The boundary 
homomorphism takes these elements of $\pi_{1}(\operatorname{Diff}(\partial M, A$ rel $R))$ to Dehn twists supported near these boundary tori. Since boundary tori are involved, we are in the Seifert-fibered case, and we can assume these Dehn twists take fibers to fibers. A non-zero element of the kernel of $\partial$ would give a non-trivial linear combination of these Dehn twists which was zero in $\mathcal{H}(M$ rel $\partial M)$. By projecting this linear combination onto $\mathcal{H}(S$ rel $\partial S)$ we see that it must be a linear combination of Dehn twists taking each fiber to itself. But by our homology interpretation of these Dehn twists, the only non-trivial combinations which could be isotopically trivial are those involving twists near all components of $\partial M$. Since we are assuming $R$ is non-empty, there are no such combinations in the image of $\partial$.

\section{Hyperbolic Manifolds}

Let $M$ be a compact connected orientable irreducible 3 -manifold with nonempty boundary. We decompose $\partial M$ into three compact subsurfaces meeting only in their boundary circles: $T$, the union of the torus components of $\partial M$; $A$, a disjoint union of annuli in the other components; and $B$, the closure of $\partial M-(A \cup T)$. We assume that all the components of $B$ have negative Euler characteristic. For brevity we write $C=A \cup T$, the "cusps" of $M$. Assume the following.

(i) $B$ and $C$ are incompressible in $M$.

(ii) Every $\pi_{1}$-injective map of a torus into $M$ is homotopic into $T$.

(iii) Every $\pi_{1}$-injective map of pairs $\left(S^{1} \times I, S^{1} \times \partial I\right) \rightarrow(M, B)$ is homotopic as a map of pairs to a map carrying $S^{1} \times I$ into either $A$ or $B$.

(iv) $M$ is not homeomorphic to $S^{1} \times S^{1} \times I$.

Note that assumptions (iii) and (iv) imply that $(M, A)$ is not of the form $(F \times I, \partial F \times I)$.

Let $\operatorname{Diff}(M, A)$ denote the diffeomorphisms of $M$ that take $A$ diffeomorphically to $A$. These also must take $M-C$ to $M-C$.

Lemma 3.1 For $M, A, T$, and $C$ as above, $M-C$ has a hyperbolic structure with totally geodesic boundary. Its group of isometries $\operatorname{Isom}(M-C)$ is finite, and $\operatorname{Isom}(M-C) \rightarrow \mathcal{H}(M, A)$ is an isomorphism.

The homomorphism $\operatorname{Isom}(M-C) \rightarrow \mathcal{H}(M, A)$ requires a bit of explanation. Each component of $C$ inherits a Euclidean structure from the corresponding 
cusp of $M-C$, and isometries of $M-C$ induce isometries of these Euclidean annuli and tori. So each isometry of $M-C$ extends uniquely to a diffeomorphism of $M$ preserving $A$.

Proof Assume first that $T=\partial M$. By a celebrated result of Thurston, $M-T$ has a complete hyperbolic structure of finite volume, and by the Mostow Rigidity Theorem $\operatorname{Isom}(M-T)$ is finite and the composition $\operatorname{Isom}(M-T) \rightarrow$ $\mathcal{H}(M) \rightarrow \operatorname{Out}\left(\pi_{1}(M)\right)$ is an isomorphism. Since $M$ is aspherical, the outer automorphism group $\operatorname{Out}\left(\pi_{1}(M)\right)$ is naturally isomorphic to the group of homotopy classes of homotopy equivalences from $M$ to $M$. Since every incompressible torus in $M$ is homotopic into $\partial M$, an application of the homotopy extension property shows that every homotopy equivalence is homotopic to one which preserves $\partial M$. By [16] and the fact that $M$ is not of the form $F \times I$, every boundary-preserving homotopy equivalence is homotopic to a diffeomorphism. Therefore $\mathcal{H}(M) \rightarrow \operatorname{Out}\left(\pi_{1}(M)\right)$ is surjective. Also by [16], it is injective and the lemma follows in the case $T=\partial M$.

Suppose now that $T \neq \partial M$. Let $N$ be the manifold obtained by identifying two copies of $M$ along $B$ (using the identity map). The boundary of $N$ is incompressible and consists of tori, and assumption (iii) ensures that every incompressible torus in $N$ is homotopic into $\partial N$. From the previous case, the interior of $N$ admits a hyperbolic structure and $\operatorname{Isom}(N-\partial N) \rightarrow \mathcal{H}(N)$ is an isomorphism. Let $\tau^{\prime}$ be the involution of $N$ that interchanges the two copies of $M$. Its fixed-point set is $B$. Let $\tau$ be the isometry in the isotopy class of $\tau^{\prime}$. Note that $\tau^{2}$ is an isometry isotopic to the identity, hence equals the identity, so $\tau$ is an involution. By [15], homotopic involutions of $N$ are strongly equivalent, ie there is a homeomorphism $k$ of $N$, isotopic to the identity, such that $k \tau^{\prime} k^{-1}=$ $\tau$. Regard $M$ as one of the copies of $M$ in $N$, so that $B$ is its frontier. Then $k(B)$ is the fixed-point set of $\tau$, and $k$ carries $M$ homeomorphically to the closure of one of the complementary components of $k(B)$. Therefore by changing coordinates using $k$ we may assume that $B$ is the fixed point set of $\tau$. The fixed-point set of an isometry is totally geodesic so the restriction to $M-C$ of the hyperbolic structure on $N-\partial N$ is complete with totally geodesic boundary.

Define $\Phi: \mathcal{H}(M, A) \rightarrow \mathcal{H}(N)$ by sending $\langle h\rangle$ to the class represented by $D(h)$, the double of $h$ along $B$. Let $\mathcal{T}$ be the subgroup of order 2 in $\mathcal{H}(N)$ generated by $\langle\tau\rangle$. We claim that $\Phi$ is injective and that $\Phi(\mathcal{H}(M, A)) \times \mathcal{T}$ is the centralizer of $\langle\tau\rangle$ in $\mathcal{H}(N)$. Suppose first that $\langle h\rangle$ lies in the kernel of $\Phi$. Let $B_{0}$ be a component of $B$. Assumption (iii) implies that $N$ does not fiber over $S^{1}$ with fiber $B_{0}$, so lemma 1.3 implies that $D(h)$ is isotopic to $1_{N}$ preserving $B_{0}$ at 
each level. Repeating for each component of $B$, we find that $D(h)$ is isotopic to the identity preserving $B$, so $\langle h\rangle$ was trivial in $\mathcal{H}(M, A)$. Clearly the image of $\Phi$ lies in the centralizer, since $D(h)$ actually commutes with $\tau$. Suppose $\langle H\rangle$ is an element in the centralizer of $\tau$. Then $H \tau H^{-1}$ is isotopic to $\tau$. Again by Tollefson's result, they must be strongly equivalent. So $H$ is isotopic to $k H$ with $k H \tau(k H)^{-1}=\tau$. This implies that $k H$ preserves $B$. If $k H$ does not reverse the sides of $B$, then it must be of the form $D(h)$, so lies in $\Phi(\mathcal{H}(M, A))$. It it does reverse the sides, then $k H \tau$ does not. If follows that $\Phi(\mathcal{H}(M, A))$ and $\mathcal{T}$ generate the centralizer. If $k H$ and $k^{\prime} H$ preserve $B$ and are isotopic, then by lemma 1.3 they are isotopic preserving $B$. Therefore it is well-defined whether an element in the centralizer of $\langle\tau\rangle$ in $\mathcal{H}(N)$ preserves the sides of $B$. In particular, elements of the image of $\Phi$ do not reverse the sides of $B$, so $\Phi(\mathcal{H}(M, A)) \cap \mathcal{T}$ consists only of the identity, and the claim follows.

From the case $T=\partial M, \operatorname{Isom}(N-\partial N) \rightarrow \mathcal{H}(N)$ is an isomorphism. An isometry on $N$ commutes with $\tau$ and preserves the sides of $B$ if and only if it is the double along $B$ of an isometry of $M$. Therefore sending an isotopy class in $\Phi(\mathcal{H}(M, A))$ to the restriction to $M$ of the unique isometry that it contains defines an inverse to $\operatorname{Isom}(M-C) \rightarrow \mathcal{H}(M, A)$.

Proposition 3.2 Let $M, B, A$, and $T$ be as above. Let $R$ be a non-empty union of components of $B, A$, and $T$, and let $R_{0}$ be the components of $R$ that have Euler characteristic zero. Then $\mathcal{H}(M, A$ rel $R) \cong H_{1}\left(R_{0} ; \mathbb{Z}\right)$, and $\pi_{i}(\operatorname{Diff}(M, A$ rel $R))=0$ for $i \geq 1$.

Proof Consider the fibration $\operatorname{Diff}(M, A$ rel $R) \rightarrow \operatorname{Diff}_{R}(M, A) \rightarrow \operatorname{diff}(R)$ where $\operatorname{diff}(R)$ is the identity component of $\operatorname{Diff}(R)$ and $\operatorname{Diff}_{R}(M, A)$ is the subgroup of $\operatorname{Diff}(M, A)$ consisting of diffeomorphisms taking each component of $R$ to itself by a diffeomorphism isotopic to the identity. This fibration gives an exact sequence:

$$
\pi_{1} \operatorname{diff}(R) \stackrel{\partial}{\longrightarrow} \mathcal{H}(M, \text { Arel } R) \longrightarrow \mathcal{H}_{R}(M, A) \longrightarrow 0
$$

The following argument shows that the map $\partial$ is injective, so $(*)$ is in fact a short exact sequence. First note that $\operatorname{diff}(R)$ is the direct product of the $\operatorname{diff}(F)$ as $F$ ranges over the components of $R$. For the components that have negative Euler characteristic, $\operatorname{diff}(F)$ is contractible, while if $F$ is a torus or annulus, $\operatorname{diff}(F)$ is homotopy equivalent to $F$. If $R_{0}$ is empty, then $\pi_{1}(\operatorname{diff}(R))$ is trivial. Otherwise, fix a component $F$ of $R_{0}$. The boundary map $\partial$ sends elements $\langle\gamma\rangle$ of $\pi_{1}(\operatorname{diff}(F))$ to Dehn twists supported in a collar neighborhood of $F$. Such a Dehn twist induces an inner automorphism of $\pi_{1}\left(M, x_{0}\right)$ for $x_{0} \in F$, 
namely, conjugation by the element of $\pi_{1}\left(M, x_{0}\right)$ represented by the loop in $F$ around which a basepoint $x_{0}$ in $F$ is carried by $\gamma$. This element uniquely determined $\langle\gamma\rangle$, in particular it is non-trivial when $\gamma$ is non-trivial. Note that Dehn twists near other components of $R_{0}$ have no effect on $\pi_{1}\left(M, x_{0}\right)$. Inner automorphisms of $\pi_{1} M$ are always non-trivial since $\pi_{1} M$ has trivial center (a standard fact about hyperbolic 3-manifolds other than the ones ruled out by assumptions (i)-(iv) above). Therefore the map $\pi_{1} \operatorname{diff}(F) \rightarrow \operatorname{Aut}\left(\pi_{1}\left(M, x_{0}\right)\right)$ is injective. Fixing basepoints $x_{1}, \ldots, x_{k}$ in the components of $R_{0}$, we obtain a composition $\pi_{1} \operatorname{diff}(R) \stackrel{\partial}{\rightarrow} \mathcal{H}(M$ rel $R) \rightarrow \prod_{i=1}^{k} A u t\left(\pi_{1}\left(M, x_{i}\right)\right)$ which is injective, showing that $\partial$ is injective.

Next we show that $\pi_{i}(\operatorname{Diff}(M, A$ rel $R))=0$ for $i \geq 1$. It is sufficient as in the proof of lemma 2.3 to check that $\partial: \pi_{1}(\operatorname{Diff}(\partial M, A$ rel $R)) \rightarrow \mathcal{H}(M$ rel $\partial M)$ is injective. Since $\pi_{1}(\operatorname{diff}(F))$ is trivial when $F$ has negative Euler characteristic, $\pi_{1}(\operatorname{Diff}(\partial M, A$ rel $R))$ is generated by elements of $\pi_{1}(\operatorname{diff}(F))$ for the torus components of $\partial M$ that are not contained in $R$. Fixing basepoints $y_{j}$ in these components, we have as before an injective homomorphism

$$
\pi_{1}\left(\operatorname{Diff}(\partial M, A \text { rel } R) \stackrel{\partial}{\longrightarrow} \mathcal{H}(M \operatorname{rel} \partial M) \rightarrow \prod A u t\left(\pi_{1}\left(M, y_{j}\right)\right),\right.
$$

showing that $\partial$ is injective.

Now we turn to the calculation of $\mathcal{H}(M, A$ rel $R)$. By lemma 3.1, we can fix a hyperbolic structure on $M-(A \cup T)$ such that $\operatorname{Isom}(M-(A \cup T))$ is finite and $\operatorname{Isom}(M-(A \cup T)) \rightarrow \mathcal{H}(M, A)$ is an isomorphism. Suppose first that $R \neq R_{0}$. Since no non-trivial isometry of a hyperbolic surface of negative Euler characteristic is isotopic to the identity, the subgroup of $\operatorname{Isom}(M-(A \cup T))$ that maps to the subgroup $\mathcal{H}_{R}(M, A)$ of $\mathcal{H}(M, A)$ is trivial if $R \neq R_{0}$, in which case $(*)$ gives $\mathcal{H}(M, A$ rel $R) \cong \pi_{1}(\operatorname{diff}(R)) \cong H_{1}\left(R_{0} ; \mathbb{Z}\right)$.

Thus we may assume that $R=R_{0}$. The subgroup $\operatorname{Isom}_{R}(M-(A \cup T)) \subset$ $\operatorname{Isom}(M-(A \cup T))$ that corresponds to the subgroup $\mathcal{H}_{R}(M, A) \subset \mathcal{H}(M, A)$ consists of isometries which on each component of $R$ are rotations isotopic to the identity. For each $\varphi_{0} \in \operatorname{Diff}(M, A$ rel $R)$ there is an isotopy $\varphi_{t}$ in $\operatorname{Diff}(M, A)$ from $\varphi_{0}$ to the isometry $\varphi_{1} \in \operatorname{Isom}_{R}(M-(A \cup T))$ corresponding to $\varphi_{0}$ under the map $\mathcal{H}(M, A$ rel $R) \rightarrow \mathcal{H}_{R}(M, A)$. The isotopy $\varphi_{t}$ is unique up to deformation since the fact that $\pi_{1} \operatorname{Diff}(M, A$ rel $R)=0$ implies that $\pi_{1} \operatorname{Diff}(M, A)=0$ by looking a few terms to the left in the sequence $(*)$.

The group of rotations of $R$ can be identified with a subspace $R^{*} \subset R$ by evaluation of rotations at a chosen basepoint in each component of $R$; in annulus components we choose the basepoint in the boundary of the annulus. The inclusion $R^{*} \hookrightarrow R$ is a homotopy equivalence. Let $G$ be the subgroup of $R^{*}$

\section{Geometry and Topology, Volume 1 (1997)}


obtained by restriction of $\operatorname{Isom}_{R}(M-(A \cup T))$. Evaluation of the path $\varphi_{t}$ at the basepoints in $R$ then gives a well-defined map $\Phi: \mathcal{H}(M, A$ rel $R) \rightarrow \pi_{1}\left(R^{*}, G\right)$ which is a homomorphism if $\pi_{1}\left(R^{*}, G\right)$ is given the group structure induced by the group structure of $R^{*}$. In fact, $\Phi$ gives a map from the short exact sequence $(*)$ to the short exact sequence $0 \rightarrow \pi_{1}(R) \rightarrow \pi_{1}\left(R^{*}, G\right) \rightarrow G \rightarrow 0$, hence $\Phi$ is an isomorphism by the five-lemma. By lifting paths to the universal cover of $R^{*}$, we identify $\pi_{1}\left(R^{*}, G\right)$ with a cocompact lattice in a Euclidean space, containing the deck transformation group $\pi_{1}(R)$ as a subgroup of finite index. Thus the group $\mathcal{H}(M, A$ rel $R) \cong \pi_{1}\left(R^{*}, G\right)$ is abstractly isomorphic to $\pi_{1}(R) \cong H_{1}(R ; \mathbb{Z})$.

Remark. The case when $A$ is empty yields the Main Theorem in the case when $M$ is a simple manifold.

\section{Decomposable Manifolds}

In this section we prove the Filtration Theorem in the general case. The Main Theorem follows immediately using lemmas 1.1 and 1.2.

Suppose first that $\partial M$ is compressible. We assume the compressible components of $\partial M$ lie in $R$. By inductive application of the Loop Theorem, one can construct a collection $E$ of finitely many disjoint properly-imbedded discs, none of which is isotopic into $\partial M$, such that each component of $M$ cut along $E$ has incompressible boundary. Note that $\pi_{0} \operatorname{Imb}(E, M$ rel $\partial E)=0$. By lemma 1.3, $\pi_{1}(\operatorname{Imb}(E, M$ rel $\partial E))=0$, so the restriction fibration

$$
\operatorname{Diff}(M \text { rel } E \cup R) \rightarrow \operatorname{Diff}(M \text { rel } R) \rightarrow \operatorname{Imb}(E, M \text { rel } \partial E)
$$

shows that $\mathcal{H}(M$ rel $R) \cong \mathcal{H}(M$ rel $E \cup R)$. The latter group can be identified with $\mathcal{H}\left(M^{\prime}\right.$ rel $\left.R^{\prime}\right)$, where $M^{\prime}$ is the result of cutting $M$ along $E$ and $R^{\prime}$ is the union of boundary components of $M^{\prime}$ corresponding to $R$. Although $M^{\prime}$ may no longer be connected, each of its components meets $R^{\prime}$. Thus we reduce to the case that $R$ is incompressible. In particular, if $M$ was a handlebody, then $\mathcal{H}(M \operatorname{rel} \partial M)$ is trivial and the proof is completed.

Assuming now that $M$ has incompressible boundary, the elementary form of the Torus-Annulus Decomposition Theorem of Jaco-Shalen and Johannson ([7, 8]) says $M$ contains a 2-dimensional submanifold $T \cup A$, where $T$ consists of incompressible tori and $A$ of incompressible annuli, such that each component $W$ of the manifold obtained by splitting $M$ along $T \cup A$ is either:

Geometry and Topology, Volume 1 (1997) 
(a) simple, meaning that $W, T_{W}$, and $A_{W}$ satisfy conditions (i)-(iv) at the beginning of section 3 , where $T_{W}$ is the union of the torus boundary components of $W$ and $A_{W}$ is the union of the components of the closure of $\partial W-\partial M$ that are annuli;

(b) an $I$-bundle over a surface of negative Euler characteristic, such that $W \cap \partial M$ is the associated $\partial I$-bundle; or

(c) Seifert-fibered, with $W \cap \partial M$ a union of fibers.

Further, when $T \cup A$ is chosen to be minimal with respect to inclusion among all such submanifolds, it is unique up to ambient isotopy of $M$. We need a relative form of this uniqueness: Two choices of $T \cup A$ having the same boundary are isotopic fixing $R$. This follows from the previous uniqueness statement since the obstruction to deforming an arbitrary isotopy to an isotopy fixing $R$ is the homotopy class of the loop of embeddings of $R \cap \partial A$ traced out during the isotopy, but $\partial A$ is disjoint from torus components of $\partial M$ (an exercise from the definitions) and there are no non-trivial loops of embedded circles in surfaces of negative Euler characteristic.

This relative uniqueness implies that the natural map $\mathcal{H}(M, T \cup A$ rel $R) \rightarrow$ $\mathcal{H}(M$ rel $R)$ is surjective. By lemma 1.3 it is also injective, so we have $\mathcal{H}(M, T \cup$ A rel $R) \cong \mathcal{H}(M$ rel $R)$. This remains true if we replace each of the annuli and tori of $T \cup A$ by two nearby parallel copies of itself, still calling the doubled collection $T \cup A$. The advantage in doing this is that now when we split $M$ along $T \cup A$, the pieces produced by the splitting are submanifolds of $M$, and $M$ is their union. The new pieces lying between parallel annuli and tori of the original $T \cup A$ we view as additional Seifert-fibered pieces.

Let $V_{1}=W_{1}$ be a piece which meets $R$, and inductively, let $V_{i}=V_{i-1} \cup W_{i}$ where $W_{i}$ is a piece which meets $V_{i-1}$, other than the pieces already in $V_{i-1}$. For completeness let $V_{0}$ be empty. Then we have restriction fibrations

$\operatorname{Diff}\left(M, T \cup A\right.$ rel $\left.V_{i} \cup R\right) \longrightarrow \operatorname{Diff}\left(M, T \cup A\right.$ rel $\left.V_{i-1} \cup R\right) \stackrel{\rho}{\longrightarrow} \operatorname{Diff}\left(W_{i}, A_{i}\right.$ rel $\left.R_{i}\right)$ where $A_{i}=W_{i} \cap A$ and $R_{i}=W_{i} \cap\left(V_{i-1} \cup R\right)$. These fibrations yield exact sequences

$0 \rightarrow \mathcal{H}\left(M, T \cup A\right.$ rel $\left.V_{i} \cup R\right) \rightarrow \mathcal{H}\left(M, T \cup A\right.$ rel $\left.V_{i-1} \cup R\right) \stackrel{\rho_{*}}{\longrightarrow} \mathcal{H}\left(W_{i}, A_{i}\right.$ rel $\left.R_{i}\right)$

where the zero at the left end is $\pi_{1}\left(\operatorname{Diff}\left(W_{i}, A_{i}\right.\right.$ rel $\left.\left.R_{i}\right)\right)$, which vanishes by lemma 2.3 or proposition 3.2. The Filtration Theorem will follow once we show that the image of each map $\rho_{*}$ has a filtration of the sort in the theorem.

The case that $W_{i}$ is hyperbolic is immediate since $\mathcal{H}\left(W_{i}, A_{i}\right.$ rel $\left.R_{i}\right)$ is finitely generated free abelian by proposition 3.2 , hence also any subgroup of it. Consider next the case that $W_{i}$ is an $I$-bundle. By lemma 2.1, $\mathcal{H}\left(W_{i}, A_{i}\right.$ rel $\left.R_{i}\right)=0$ 
unless $R_{i} \subset A_{i}$. In the latter case $\mathcal{H}\left(W_{i}, A_{i}\right.$ rel $\left.R_{i}\right) \cong \mathcal{H}(S$ rel $p(R))$ with $p(R)$ a union of components of $\partial S$. The image of $\rho_{*}$ has finite index in this group since it contains the finite-index subgroup represented by diffeomorphisms of $W_{i}$ which restrict to the identity on $A_{i}$, corresponding to elements of $\mathcal{H}(S$ rel $p(R))$ represented by diffeomorphisms which are the identity on $\partial S$.

There remains the case that $W_{i}$ is Seifert-fibered. By lemma 2.2, there is a short exact sequence

$$
\begin{gathered}
0 \longrightarrow H_{1}\left(S, \partial S-p\left(R_{i}\right) ; \mathbb{Z}\right) \longrightarrow \mathcal{H}\left(W_{i}, A_{i} \text { rel } R_{i}\right) \longrightarrow \\
\mathcal{H}^{*}\left(S, E \cup p\left(A_{i}\right) \operatorname{rel} p\left(R_{i}\right)\right) \longrightarrow 0 .
\end{gathered}
$$

As we noted in the Remark at the end of section $2, \mathcal{H}^{*}\left(S, E \cup p\left(A_{i}\right)\right.$ rel $\left.p\left(R_{i}\right)\right)$ is a subgroup of finite index in a mapping class group $\mathcal{H}\left(S_{0}, p\left(A_{i}\right)\right.$ rel $\left.p\left(R_{i}\right)\right)$. The image of $\rho_{*}$ projects into $\mathcal{H}^{*}\left(S, E \cup p\left(A_{i}\right)\right.$ rel $\left.p\left(R_{i}\right)\right)$ as a subgroup of finite index, since the image of $\rho_{*}$ contains the isotopy classes represented by diffeomorphisms which are the identity on $\partial W_{i}$. Since $R_{i}$ is not empty, $H_{1}\left(S, \partial S-p\left(R_{i}\right) ; \mathbb{Z}\right)$ is free abelian. So it intersects the image of $\rho_{*}$ in a free abelian group, and the proof is complete.

\section{References}

[1] A Gramain, Le type d'homotopie du groupe des difféomorphismes d'une surface compacte, Ann. École Norm. Sup. 6 (1973) 53-66

[2] A Hatcher, A proof of the Smale conjecture, Ann. of Math. 117 (1983) 553-607

[3] A Hatcher, Homeomorphisms of sufficiently large $P^{2}$-irreducible 3-manifolds, Topology 15 (1976) 343-347

[4] A Hatcher, On triangulations of surfaces, Topology Appl. 40 (1991) 189-194

[5] W Heil, J Tollefson, On Nielsen's theorem for 3-manifolds, Yokohama Math. J. 35 (1987) 1-20

[6] N V Ivanov, Diffeomorphism groups of Waldhausen manifolds, J. Soviet Math. 12 (1979), 115-118 (Russian original in Zap. Nauk. Sem. Leningrad Otdel. Mat. Inst. Steklov 66 (1976) 172-176. Detailed write-up: Spaces of surfaces in Waldhausen manifolds, Preprint LOMI P-5-80 Leningrad (1980))

[7] W Jaco, P Shalen, Seifert fibered spaces in 3-manifolds, Mem. Amer. Math. Soc. 220 (1979)

[8] K Johannson, Homotopy equivalences of 3-manifolds with boundaries, Springer-Verlag Lecture Notes in Mathematics 761 (1979)

[9] F Laudenbach, Topologie de dimension trois. Homotopie et isotopie, Astérisque 12 (1974) 11-152

Geometry and Topology, Volume 1 (1997) 
[10] J Kalliongis, D McCullough, Isotopies of 3-manifolds, Topology Appl. 71 (1996) 227-263

[11] J Kalliongis, D McCullough, Fiber-preserving imbeddings and diffeomorphisms, preprint

[12] R Kirby, Problems in low-dimensional topology, in Geometric Topology Part II, ed W Kazez, AMS/IP Studies in Advanced Mathematics Vol. 2.2 (1997) 35-473

[13] D McCullough, Virtually geometrically finite mapping class groups of 3manifolds, J. Diff. Geom. 33 (1991) 1-65

[14] J-P Serre, Cohomologie des groupes discrets, in Prospects in Mathematics, Annals of Mathematics Study No. 70 (1971) 77-169

[15] J Tollefson, Involutions of sufficiently large 3-manifolds, Topology 20 (1981), 323-352

[16] F Waldhausen, On irreducible 3-manifolds which are sufficiently large, Ann. of Math. 87 (1968) 56-88

[17] F Waldhausen, Recent results on sufficiently large 3-manifolds, Proceedings of Symposia in Pure Mathematics 32 (1978) 21-38 